\title{
Transforaminal Endoscopic Partial Vertebrectomy for Decompression of Spinal Metastases: A Novel, Minimally Invasive Approach to Treating Symptomatic Lumbar Vertebral Body Metastases
}

\author{
Michael Brooks ${ }^{1,2}$, Yingda $\mathrm{Li}^{3,4}$ \\ ${ }^{1}$ Department of Neurosurgery, Liverpool Hospital, Liverpool, NSW, Australia \\ ${ }^{2}$ South West Sydney Clinical School, School of Medicine, University of New South Wales (UNSW), Liverpool, NSW, Australia \\ ${ }^{3}$ Department of Neurosurgery, Westmead Hospital, Westmead, NSW, Australia \\ ${ }^{4}$ Sydney Medical School, University of Sydney, Camperdown NSW, Australia
}

Received: September 6, 2021

Revised: September 28, 2021

Accepted: October 3, 2021

Corresponding Author:

Michael Brooks, MD

Department of Neurosurgery,

Liverpool Hospital, Elizabeth Street,

Liverpool, NSW 2170, Australia

Tel: +61-4-15-184-123

Fax: +61-2-8738-3343

E-mail:mrbrooks@outlook.com.au

\begin{abstract}
Vertebral body metastases cause a significant degree of disability in patients with advanced oncological disease. Surgical treatment is an effective treatment however it comes with a long recovery period and significant post-operative pain. When spinal metastases are involved, wound healing is a limiting factor in recommencement of radiotherapy. An endoscopic vertebrectomy allows for safe rhizolysis and subsequent fixation to maintain stability. We present the first case of endoscopic partial vertebrectomy for symptomatic spinal metastases. A fifty-eight-year-old lady presented with spinal metastases secondary to metastatic lung cancer. She underwent a transforaminal endoscopic partial vertebrectomy with rhizolysis of the exiting nerve root and percutaneous pedicle screw fixation of the L3-L5 vertebral bodies. She recovered well post-operatively with complete resolution of the radicular pain and a return to baseline mobility. Endoscopic partial vertebrectomy presents a new approach to treatment of spinal metastases. This allows for a reduction in post-operative length of hospital stay, post-operative pain and an improved rate of wound healing. The reduction in wound healing allows for a faster return to treatment when compared with an open approach. An endoscopic approach is beneficial for treatment of foraminal pathology, allowing for good visualisation of the nerve root to perform optimal rhizolysis.
\end{abstract}

Key Words: Endoscopy, Minimally invasive surgical procedures, Spine

\section{INTRODUCTION}

Spinal metastases cause a significant degree of morbidity in cancer patients. Foraminal deposits of cancer can compress the exiting nerve root causing severe radiculopathic pain. Surgical management has classically included open debulking and instrumentation of adjacent levels ensuring spinal stability [1].
This improves symptoms and quality of life, however can have a long recovery period with significant operative site pain, and limitations on normal activities.

Minimally invasive spine surgery (MISS) has presented an alternate approach to open cases. This approach allows for smaller incision sites with less damage to the normal tissue, improving wound healing times [2], with a lower rate of wound

Copyright @ 2021 Korean Minimally Invasive Spine Surgery Society

This is an Open Access article distributed under the terms of the Creative Commons Attribution Non-Commercial License (http://creativecommons.org/licenses/by-nc/4.0/) which permits unrestricted non-commercial use, distribution, and reproduction in any medium, provided the original work is properly cited. 
dehiscence. In lumbar fusion cases, this results in less intra-operative blood loss and a lower length of stay post-operatively [3]. This is particularly important in patients with metastatic cancer for many reasons, potentially reducing the recovery time and allowing for commencement of radiotherapy and systemic therapy at an earlier time due to quicker wound healing times.

Endoscopic spine surgery represents the least invasive of all MISS approaches. To the knowledge of the authors, no cases have been published wherein an endoscopic partial vertebrectomy has been used to treat symptomatic vertebral body metastasis.

\section{CASE REPORT}

\section{History}

A 58-year-old female presented with a ten-week history of lower back pain with a new right-sided L4 radiculopathy and associated sensory changes. She has a known background of metastatic non-small cell lung cancer (NSCLC) with L4 vertebral body metastasis, previously treated with radiotherapy. Following a recent fall, her mobility had deteriorated requiring assistance for mobilisation.

\section{Examination}

She presented with an antalgic gait affecting her right leg. Her power was $5 / 5$ over all lower limb myotomes and normal reflexes. She had a slight reduction to pinprick sensation over the right L4 dermatome. Her examination was otherwise normal. She had a sacral scar from a spinal cord stimulator for post-operative pain following a hysterectomy which precluded MRI.

Her CT scan demonstrated a pathological fracture of the right postero-inferior corner of the L4 vertebral body associated with a lytic metastasis. There was a retropulsed fragment extending into the right $\mathrm{L} 4 / 5$ foramen causing foraminal stenosis (Figure 1 ).

The decision was made to pursue operative management of her radicular symptoms. Owing to the palliative nature of her metastatic NSCLC, the decision was made to use a MISS approach to facilitate rapid recovery.

\section{Operation}

Trajectory was planned off the preoperative CT through Kambin's triangle, past the superior articular facet of L5 and docking on the L4/5 disc space (Figure 1). The patient was prone on a Jackson table. Percutaneous targeting needle was inserted to the superior articular facet then 'walked' down onto the $L 4 / 5$ annulus under fluoroscopic control and intraoperative neuromonitoring with subsequent sequential dilators to allow for endoscope insertion (TESSYS, Joimax Inc., Irvine CA 92618, USA). L4-5 annulotomy using endoscopic rongeurs to create a ledge. Subsequent partial vertebrectomy under fluoroscopic guidance with the shaft of the rongeur used to deflect the exiting nerve root (Figure 2). After removal of the retropulsed fragment, the foraminotomy was extended to the residual superior articular process, with removal of the remaining ligamentum flavum to allow for sufficient neurolysis (Figure 3).

Once sufficient decompression was obtained, percutaneous pedicle screw fixation was performed. Bilateral cemented screws were placed in L3 and L5, with a single non-cemented screw on the left side of L4. Post-operative CT scan confirmed
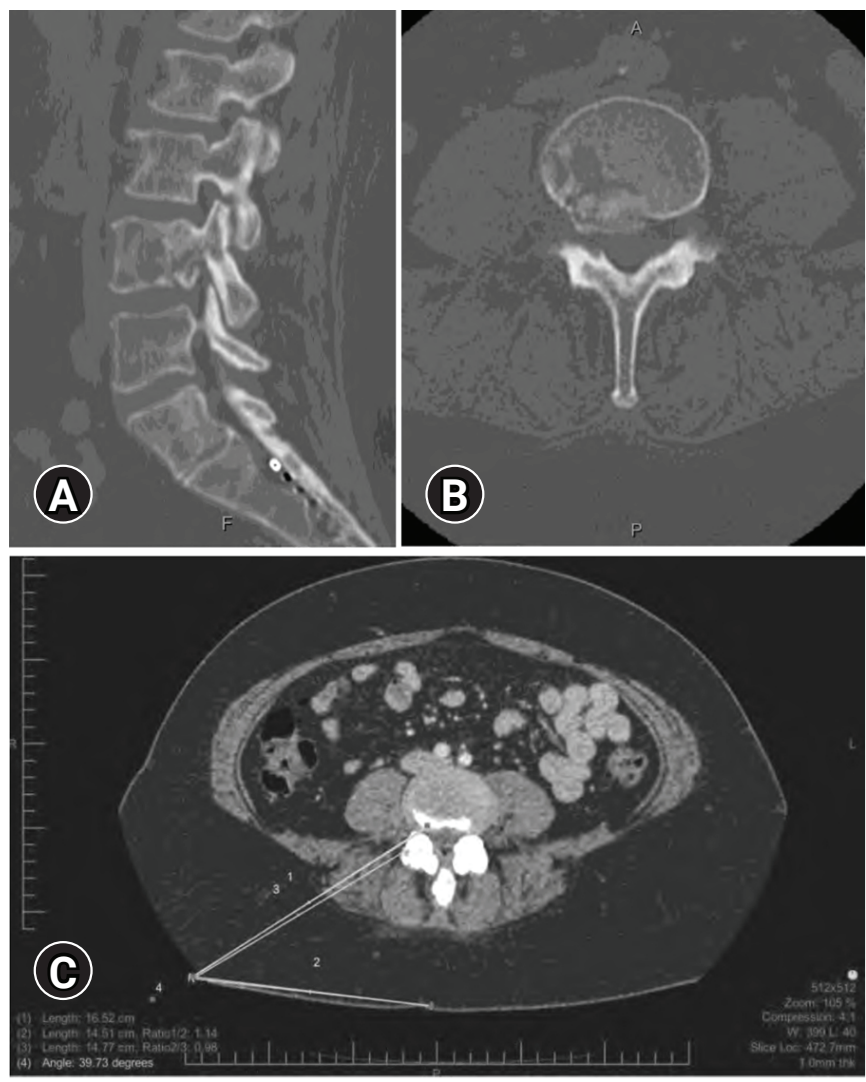

Figure 1. (A) Sagittal CT scan taken pre-operatively demonstrating a posteroinferior $L 4$ vertebral body fracture in the region of a Iytic metastatic deposit. Note the presence of transitional anatomy of $L 5$ and the sacral stimulator leads. (B) Corresponding axial slice showing the retropulsed fragment into the right $L 4 / 5$ neural foramen. (C) Pre-operative planning scan. Line 1 represents the ideal trajectory through Kambin's triangle. Line 2 represents the distance from the midline to derive angle 4 . Line 3 represents the depth at which the percutaneous targeting needle is expected to hit bone. 

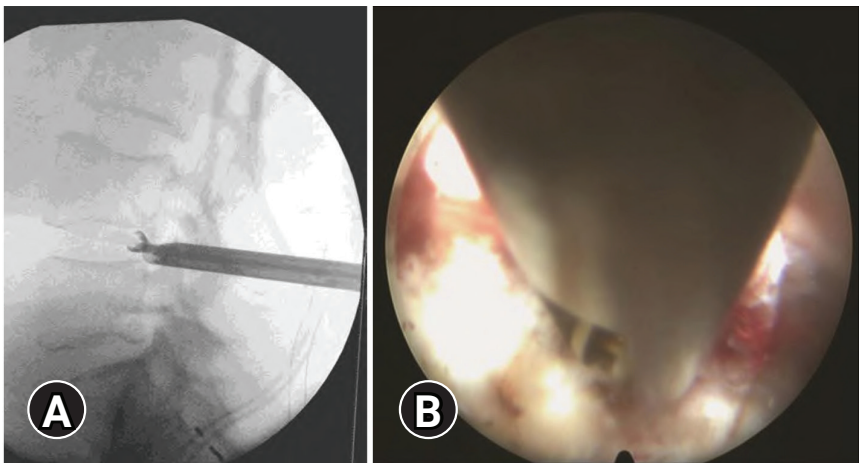

Figure 2. (A) Intra-operative fluoroscopic view of rongeurs in both disc and vertebral body to create the cavity and excise bone fragments. (B) Intra-operative endoscopic view demonstrating the shaft of the rongeur deflecting the nerve root (NR).
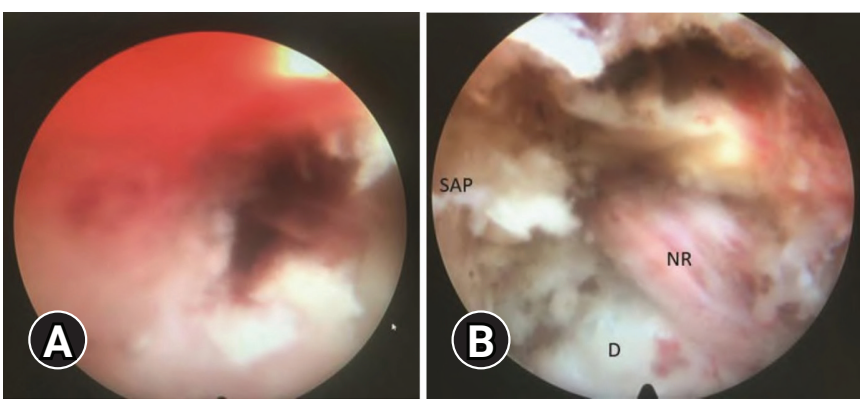

Figure 3. (A) Endoscopic view of the vertebral cavity. (B) Endoscopic view of the decompressed nerve root (NR). Disc (D) and remnant superior articular process (SAP) labelled for orientation.

adequate decompression of the foramen and well-seated hardware (Figure 4).

At follow up four months post-op her radiculopathic pain had completely resolved but some residual numbness remained. Post-operative X-Ray demonstrated stable alignment of the vertebrae (Figure 5).

\section{Ethical Statement}

The patient consented to this report being written and consented to undergo the procedure.

\section{DISCUSSION}

Spinal metastasis is a common feature of certain cancers such as prostate and lung. When bony deposits occur, tumour growth can cause localised pain due to bony destruction and compression of the spinal cord and neural elements, including the exiting nerve root. Partial and complete vertebrectomy have been shown to prevent further deterioration in patients with
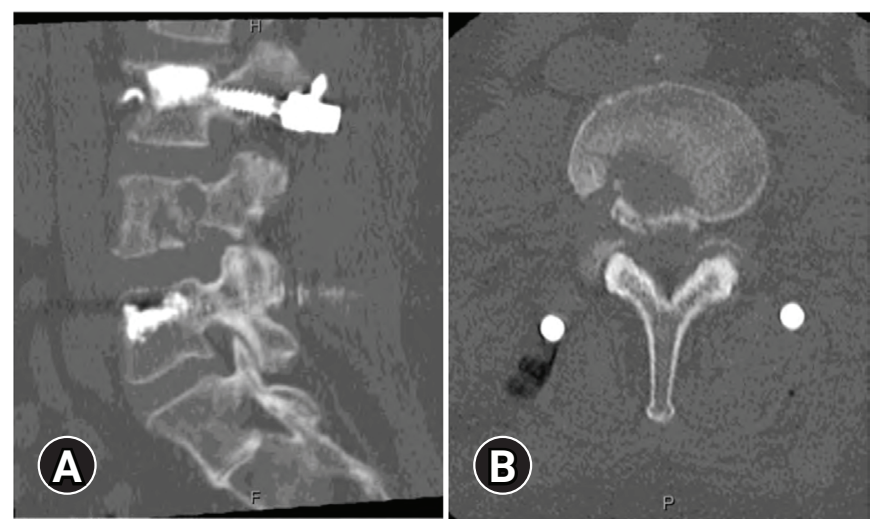

Figure 4. (A) Post-operative CT scan with sagittal slice demonstrating a good degree of decompression of the neural foramen. (B) Axial slice demonstrating the channel used to successfully complete the partial vertebrectomy.

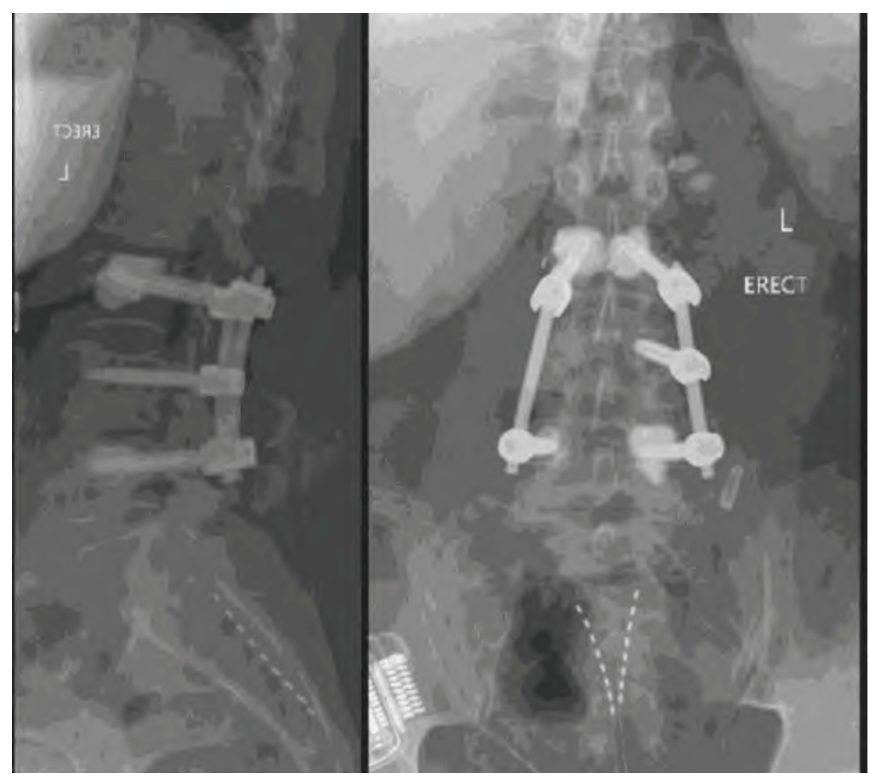

Figure 5. X-ray taken at 6 weeks post-operatively demonstrating stable vertebral body alignment and appropriate hardware placement.

symptomatic vertebral disease [4].

With the advent of MISS, surgical outcomes are improving, and many procedures are being optimised endoscopically. Early papers investigated benefits of endoscopic discectomy [5] and foraminotomy [6], which expanded over time to include spinal fusion $[7,8]$. Many benefits have been deduced from MISS approaches to spinal fusion, including reduced blood loss, decreased operative time, and decreased wound complications while maintaining similar outcomes for pain reduction and disability [9]. Finally, total recovery time has been demonstrated to be significantly better in terms of length of hospital 
stay [10] and time to return to work [11].

Benefits of MISS approaches are enhanced in patients with spinal metastases, where treatment is often palliative, due to life-limiting disease. Despite this, surgery has been demonstrated to have significant benefits in pain reduction and arrestation of mobility decline [12]. MISS has a strong potential to provide additional benefits to this due to the propensity towards reduced hospital stay and time to return to work. In palliative patients, a MISS approach can reduce their recovery time and further benefit their quality of life. It is within this select group of vulnerable patients that endoscopic spine surgery, which represents the least invasive of all MISS approaches, may be potentially transformative.

Multiple approaches have been used in endoscopic approaches to lumbar spinal pathology. We elected to use a transforaminal endoscopic approach as it is well suited to treatment of pathologies requiring access to the foramen through Kambin's triangle [13], a natural anatomic corridor not otherwise reachable through traditional approaches without violation of native bony structures and disruption of the posterior tension band and paraspinal musculature. This approach provides good access to the intervertebral disc, allowing for highly effective decompression of the neural foramen due [14]. Currently, it has not been used for debulking of spinal metastases compressing the exiting nerve root, perhaps due to limitations in achieving adequate bony removal and haemostasis, and its inherently steep learning curve. The approach we presented allowed for effective removal of the lesion and decompression of the exiting nerve root.

\section{CONCLUSION}

This demonstrates the effectiveness of an endoscopic approach to treating symptomatic spinal metastases in a patient with known metastatic NSCLC. The endoscopic approach allows for adequate decompression of the nerve root while minimising post-operative pain.

\section{CONFLICT OF INTEREST}

No potential conflict of interest relevant to this article.

\section{REFERENCES}

1. Klimo P Jr, Schmidt MH. Surgical management of spinal metastases. Oncologist 2004;9:188-196.

2. Eck JC, Hodges S, Humphreys SC. Minimally invasive lumbar spinal fusion. J Am Acad Orthop Surg 2007;15:321-329.

3. McGirt MJ, Parker SL, Mummaneni P, Knightly J, Pfortmiller D, Foley $\mathrm{K}$, et al. Is the use of minimally invasive fusion technologies associated with improved outcomes after elective interbody lumbar fusion? Analysis of a nationwide prospective patient-reported outcomes registry. Spine J 2017;17:922-932.

4. Roser S, Maharaj MM, Taylor MA, Kuru R, Hansen MA, Ferch R. Vertebrectomy in metastatic spinal tumours: a 10 year, single-centre review of outcomes and survival. J Clin Neurosci 2019;68:218-223.

5. Obenchain TG. Laparoscopic lumbar discectomy: case report. J Laparoendosc Surg 1991;1:145-149.

6. Fessler RG, Khoo LT. Minimally invasive cervical microendoscopic foraminotomy: an initial clinical experience. Neurosurgery 2002;51:S37-S45.

7. Brusko GD, Wang MY. Endoscopic lumbar interbody fusion. Neurosurg Clin N Am 2020;31:17-24.

8. Foley KT, Gupta SK, Justis JR, Sherman MC. Percutaneous pedicle screw fixation of the lumbar spine. Neurosurg Focus 2001;10:E10.

9. Mobbs RJ, Sivabalan P, Li J. Technique, challenges and indications for percutaneous pedicle screw fixation. J Clin Neurosci 2011;18:741-749.

10. Adogwa O, Parker SL, Bydon A, Cheng J, McGirt MJ. Comparative effectiveness of minimally invasive versus open transforaminal lumbar interbody fusion: 2-year assessment of narcotic use, return to work, disability, and quality of life. J Spinal Disord Tech 2011;24:479-484.

11. Chang HK, Huang M, Wu JC, Huang WC, Wang MY. Less opioid consumption with enhanced recovery after surgery transforaminal lumbar interbody fusion (TLIF): a comparison to standard minimally-invasive TLIF. Neurospine 2020;17:228-236.

12. Kan P, Schmidt MH. Minimally invasive thoracoscopic approach for anterior decompression and stabilization of metastatic spine disease. Neurosurg Focus 2008;25:E8.

13. Choi G, Pophale CS, Patel B, Uniyal P. Endoscopic spine surgery. J Korean Neurosurg Soc 2017;60:485-497.

14. Gibson JNA, Subramanian AS, Scott CEH. A randomised controlled trial of transforaminal endoscopic discectomy vs microdiscectomy. Eur Spine J 2017;26:847-856. 